\title{
Lenvatinib plus everolimus or pembrolizumab versus sunitinib in advanced renal cell carcinoma: study design and rationale
}

\author{
Viktor Grünwald*,1, Thomas Powles², Toni K Choueiri ${ }^{3}$, Thomas E Hutson ${ }^{4}$, Camillo Porta ${ }^{5}$, \\ Masatoshi Eto ${ }^{6}$, Cora N Sternberg ${ }^{7}$, Sun Young Rha ${ }^{8}$, Cixin $\mathrm{S} \mathrm{He}^{9}$, Corina E Dutcus ${ }^{9}$, Alan \\ Smith $^{9}$, Lea Dutta ${ }^{9}$, Kalgi Mody ${ }^{9}$ \& Robert J Motzer ${ }^{10}$ \\ ${ }^{1}$ Clinic for Hematology, Hemostasis, Oncology and Stem Cell Transplantation, University Hospital Essen, Essen, Germany \\ ${ }^{2}$ Experimental Cancer Medicine, Barts Cancer Institute, London, UK \\ ${ }^{3}$ Lank Center for Genitourinary Oncology, Dana-Farber Cancer Institute, Boston, MA, USA \\ ${ }^{4}$ Urologic Oncology Program, Baylor University Medical Center, Dallas, TX, USA \\ ${ }^{5}$ University of Pavia \& Division of Translational Oncology, IRCCS Istituti Clinici Scientifici Maugeri, Pavia, Italy \\ ${ }^{6}$ Department of Urology, Kyushu University, Fukuoka, Japan \\ ${ }^{7}$ Weill Cornell Medicine, New York-Presbyterian, New York, NY, USA \\ ${ }^{8}$ Yonsei Cancer Center, Yonsei University College of Medicine, Seoul, South Korea \\ ${ }^{9}$ Eisai Inc., Woodcliff Lake, NJ, USA \\ ${ }^{10}$ Department of Medicine, Memorial Sloan Kettering Cancer Center, New York, NY, USA \\ *Author for correspondence: Tel.: +49 201723 85584; Viktor.Gruenwald@uk-essen.de
}

Aim: Lenvatinib plus everolimus is approved for the treatment of advanced renal cell carcinoma (RCC) after one prior vascular endothelial growth factor-targeted therapy. Lenvatinib plus pembrolizumab demonstrated promising antitumor activity in a Phase I/II trial of RCC. Methods: We describe the rationale and design of the CLEAR study, a three-arm Phase III trial comparing lenvatinib plus everolimus and lenvatinib plus pembrolizumab versus sunitinib monotherapy for first-line treatment of RCC. Eligible patients must have advanced clear cell RCC and must not have received any prior systemic anticancer therapy. The primary end point is progression-free survival; secondary end points include objective response rate, overall survival, safety, health-related quality of life and pharmacokinetics. Biomarker evaluations are included as exploratory end points.

First draft submitted: 28 September 2018; Accepted for publication: 17 December 2018; Published online: 28 January 2019

Keywords: everolimus $\bullet$ first-line treatment $\bullet$ immuno-oncology $\bullet$ lenvatinib $\bullet$ pembrolizumab $\bullet$ renal cell carcinoma - sunitinib • tyrosine kinase inhibitor

Cancers of the kidney and renal pelvis are among the most common cancers worldwide [1]. Regions with the highest estimated incidence rates of kidney cancer include North America, Australia/New Zealand and Europe, with incidence rates much lower in most parts of Africa and Asia [2]. It is the eighth most common cancer in the USA, with 65,340 new diagnoses and 14,970 deaths estimated for 2018 [3]. The most common type of kidney cancer is renal cell carcinoma (RCC), comprising approximately $85 \%$ of renal malignancies [4]. The 5 -year survival for European patients who were diagnosed between 2000 and 2007 is reported as $60.6 \%$, compared with $72.4 \%$ for the US population [5].

Angiogenesis is known to be integral to the development of clear cell RCC [6], and as such, several pathways involved in angiogenesis have been important therapeutic targets. A major component of the angiogenic process in RCC is VEGF [7], which has been a mainstay of anti-angiogenic therapies. Another pathway involved in the development of RCC is mediated by the mammalian target of rapamycin (mTOR), which is downstream of PI3K and protein kinase $\mathrm{B}$ and is regulated by $P T E N$ [6-8]. Inhibition of the $\mathrm{mTOR}$ pathway can inhibit both angiogenesis and tumor cell proliferation $[6,8]$. The FGF pathway also plays an important role in angiogenesis in RCC, promoting

Future Medicine 
vascularization, lymphangiogenesis and cellular growth [9,10]. In RCC, increased plasma concentrations of FGF have been shown to correlate with high tumor grade and stage, metastasis and poor prognosis [11-13].

In addition to angiogenesis and tumor cell growth, exploration of immunological mechanisms has led to the relatively recent identification of the role of different immune checkpoints (e.g., cytotoxic T-lymphocyte associated protein- 4 and PD-1) and their role in circumventing the body's antitumor immune response in RCC [14,15]. The PD-1 signaling appears to be an important regulator of tumor immune tolerance in RCC, with increased expression of PD-L1 correlating with poorer survival [14,15]. Interaction of PD-L1 with its receptor PD-1 (expressed on CD4 ${ }^{+}$ and $\mathrm{CD}^{+} \mathrm{T}$ cells, dendritic cells, B cells and natural killer cells) attenuates the antitumor immune response by inhibiting $\mathrm{T}$ cell function via induction of apoptosis, inhibition of cytokine release and decreased clonal expansion of $\mathrm{T}$ cells [14].

Overall, the measurement of PD-L1 expression by immunohistochemistry to predict response to immunooncology therapy in patients with RCC has been confounded due to differences in assays, expression cut-off levels, and the definition of positive (e.g., tumor vs immune) cells [16]. In a Phase III trial comparing nivolumab with everolimus in patients with previously treated advanced RCC (aRCC; CheckMate 025; ClinicalTrials.gov, number NCT01668784), nivolumab improved overall survival (OS) compared with everolimus, irrespective of PD-L1 expression [17]. In a Phase III trial comparing nivolumab plus ipilimumab with sunitinib in patients with previously untreated advanced clear cell RCC (CheckMate 214; ClinicalTrials.gov, number NCT02231749), median progression-free survival (PFS) was improved among patients with $\geq 1 \%$ PD-L1 expression who were treated with nivolumab plus ipilimumab (22.8 months) compared with sunitinib (5.9 months; disease progression or death hazard ratio [HR]: 0.46; 95\% CI: 0.31-0.67), whereas median PFS was not improved among patients with $<1 \%$ PD-L1 expression who were treated with nivolumab plus ipilimumab (11.0 months) compared with sunitinib (10.4 months; disease progression or death HR: 1.00; 95\% CI: 0.80-1.26) [18]. However, nivolumab plus ipilimumab therapy improved OS compared with sunitinib, irrespective of PD-L1 expression in patients with intermediate or high risk $[16,18]$. Thus, the predictive role of PD-L1 in aRCC is not entirely clear and may vary between lines of treatment.

The current treatment approach for patients with aRCC consists of sequential administration of single-agent tyrosine kinase inhibitor (TKI) therapies that target either the VEGF, mTOR or PD-1 pathways [19,20]. Until recently, very few agents proved able to increase OS; despite this, the OS of patients with aRCC has improved over time using sequential administration of single-agent therapy [21]. First-line therapy typically consists of treatment with anti-VEGF agents, typically sunitinib or pazopanib $[19,20]$. In 2017 , in a randomized, Phase II trial, cabozatinib demonstrated superior efficacy compared with sunitinib as first-line therapy for patients with aRCC of intermediate or high risk [22]. In addition, the combination of ipilimumab and nivolumab was approved by the US FDA for the treatment of treatment-naive, intermediate- or poor-risk aRCC in April 2018 [23].

However, existing first-line therapies for RCC are unsatisfactory due to toxicities and the development of drug resistance [24]. Approximately $20-30 \%$ of patients do not respond to initial therapy and progress within $\leq 3$ months, indicating primary or intrinsic resistance to molecular-targeted agents [25-30]. Angiogenic escape due to activation of VEGF-independent pathways such as FGF-associated signaling is a major mechanism of such resistance $[27,29,31,32]$. Optimization of efficacy may be achieved by combining therapeutic agents that have either broader or distinct mechanisms of action. It is thought that the combination of anti-angiogenic agents with mTOR inhibitors may overcome alternative resistance pathways [29].

Until recently, results of most combination-therapy studies (i.e., temsirolimus plus bevacizumab, temsirolimus plus sunitinib, erlotinib plus bevacizumab, sunitinib plus bevacizumab, everolimus plus bevacizumab, everolimus plus sunitinib, everolimus plus dovitinib) have shown no survival advantage over monotherapy with approved single agents and, in some cases, an unacceptably high degree of toxicity [33-40]. Notable exceptions included the successful combination of nivolumab plus ipilimumab for the treatment of intermediate- or poor-risk, previously untreated, aRCC [23], and lenvatinib plus everolimus for the treatment of aRCC following one prior VEGF-therapy [41]. More recently, atezolizumab plus bevacizumab was reported to demonstrate longer PFS versus sunitinib in patients with PD-L1 ${ }^{+}$metastatic RCC [42]. The Phase III JAVELIN trial of avelumab plus axitinib also recently reported improved PFS in patients with aRCC irrespective of PD-L1 status [43]. Two other relevant clinical trials of combination therapies are currently underway in aRCC - axitinib plus pembrolizumab, which recently reported improved OS and PFS outcomes versus sunitinib monotherapy (KEYNOTE-426; NCT02853331 [44,45]), and cabozantinib plus nivolumab (CheckMate 9ER; NCT03141177 [46]), indicative of the high degree of interest 
and promise held for these combination therapy strategies in this disease space with TKIs, which inhibit VEGF receptors plus immune checkpoint inhibitors.

\section{Lenvatinib}

Lenvatinib is a potent multitargeted receptor TKI that selectively inhibits VEGF receptors (VEGFR1, VEGFR2, VEGFR3) in addition to other pro-angiogenic and oncogenic pathway-related tyrosine kinases, including FGFR 1-4, PDGFR $\alpha$, KIT and RET [47-49]. Lenvatinib inhibited VEGF-driven VEGFR2 phosphorylation and suppressed proliferation and tube formation in human umbilical vein endothelial cell models [47,50,51]. Lenvatinib also inhibited FGF-driven tube formation in human umbilical vein endothelial cell models [52] and inhibited VEGF- and FGF-induced angiogenesis in preclinical models [50]. The antitumor activity of lenvatinib in vivo has been shown in numerous xenograft animals $[47,50,51,53]$.

Clinical evidence of the antitumor activity of lenvatinib in aRCC was first provided in an open-label, Phase I, dose-escalation study in patients with advanced solid tumors [54]. Among all the tumor types studied, lenvatinib appeared to be particularly active in RCC, with four of nine patients with RCC achieving a partial response, out of a total of seven partial responses overall [54].

\section{Lenvatinib plus everolimus}

In a mouse xenograft model, the combination of lenvatinib and everolimus demonstrated superior antiangiogenic and antitumor activity compared with each single agent alone [51]. However, in another xenograft model, the combination regimen demonstrated enhanced antitumor activity but similar antiangiogenic activity to that of each monotherapy, suggesting that other mechanisms distinct from angiogenesis may underlie the observed antitumor activity of lenvatinib plus everolimus [51]. The mechanism of action of the combination of lenvatinib and everolimus (Figure 1A [53]) was further investigated in cell-based nonclinical models, and it is hypothesized that the dual inhibition of the VEGF- and FGF-driven mitogen-activated protein kinase and mTOR pathways in endothelial cells may contribute to the enhanced antiangiogenic activity of the combination treatment [51]. In addition, dual targeting of the mTOR-S6K-S6 pathway by the lenvatinib plus everolimus combination may contribute toward the superior antitumor activity of the combination [51].

A randomized, open-label, Phase Ib study in 20 patients with metastatic RCC established the maximum tolerated dose and recommended Phase II dose as lenvatinib $18 \mathrm{mg}$ once daily in combination with everolimus $5 \mathrm{mg}$ once daily [56]. In an open-label, randomized, Phase II study, lenvatinib plus everolimus and lenvatinib alone resulted in a PFS benefit over everolimus alone (HR: 0.40; 95\% CI: 0.24-0.68; $\mathrm{p}=0.0005$ and HR: $0.61 ; 95 \%$ CI: $0.38-0.98 ; \mathrm{p}=0.048$, respectively) in patients with advanced or metastatic RCC and disease progression after one previous VEGF-targeted therapy [41]. The combination of lenvatinib plus everolimus also significantly prolonged OS compared with single-agent everolimus (HR: 0.51; 95\% CI: 0.30-0.88; $\mathrm{p}=0.024$ ) [41]. Based on the results of the Phase II study [41], lenvatinib in combination with everolimus was designated as a breakthrough therapy by the US FDA in July 2015 [57]. The combination was subsequently approved in various countries, including the US and those in Europe, for patients with aRCC following one prior anti-angiogenic therapy [58,59]. An ongoing Phase II study (NCT03173560) is investigating whether a lower dose of lenvatinib can demonstrate the same efficacy as that observed in the Phase II trial, which led to the approval of the combination but with reduced toxicity [60]. Patients with clear cell RCC, who had disease progression following one VEGF-targeted therapy, will be randomized $1: 1$ to receive either lenvatinib $18 \mathrm{mg} /$ day plus everolimus $5 \mathrm{mg} /$ day or lenvatinib $14 \mathrm{mg}$ /day plus everolimus $5 \mathrm{mg} /$ day [60].

\section{Lenvatinib plus pembrolizumab}

In addition to its antitumor and antiangiogenic effects, VEGF-targeted treatments may also act, in part, by preventing VEGF-mediated immune suppression [61]. In syngeneic mouse models, the combination of lenvatinib with monoclonal antibodies against PD-1 resulted in greater antitumor activity than either agent alone and was accompanied by an improved immune response, likely mediated by $\mathrm{CD} 8^{+} \mathrm{T}$ cells [62]. Lenvatinib treatment also significantly decreased the population of tumor-associated macrophages in tumors, which enhanced the activity of PD-1 signal inhibitors (Figure 1B) [62]. Additionally, treatment with lenvatinib has been shown to inhibit TH2 and enhance TH1 immune response, leading to the activation of memory T cells [63]. Taken together, these preclinical studies demonstrating lenvatinib's immunomodulatory activity provide mechanistic rationale for the study of lenvatinib in combination with an anti-PD-1 agent such as pembrolizumab. 


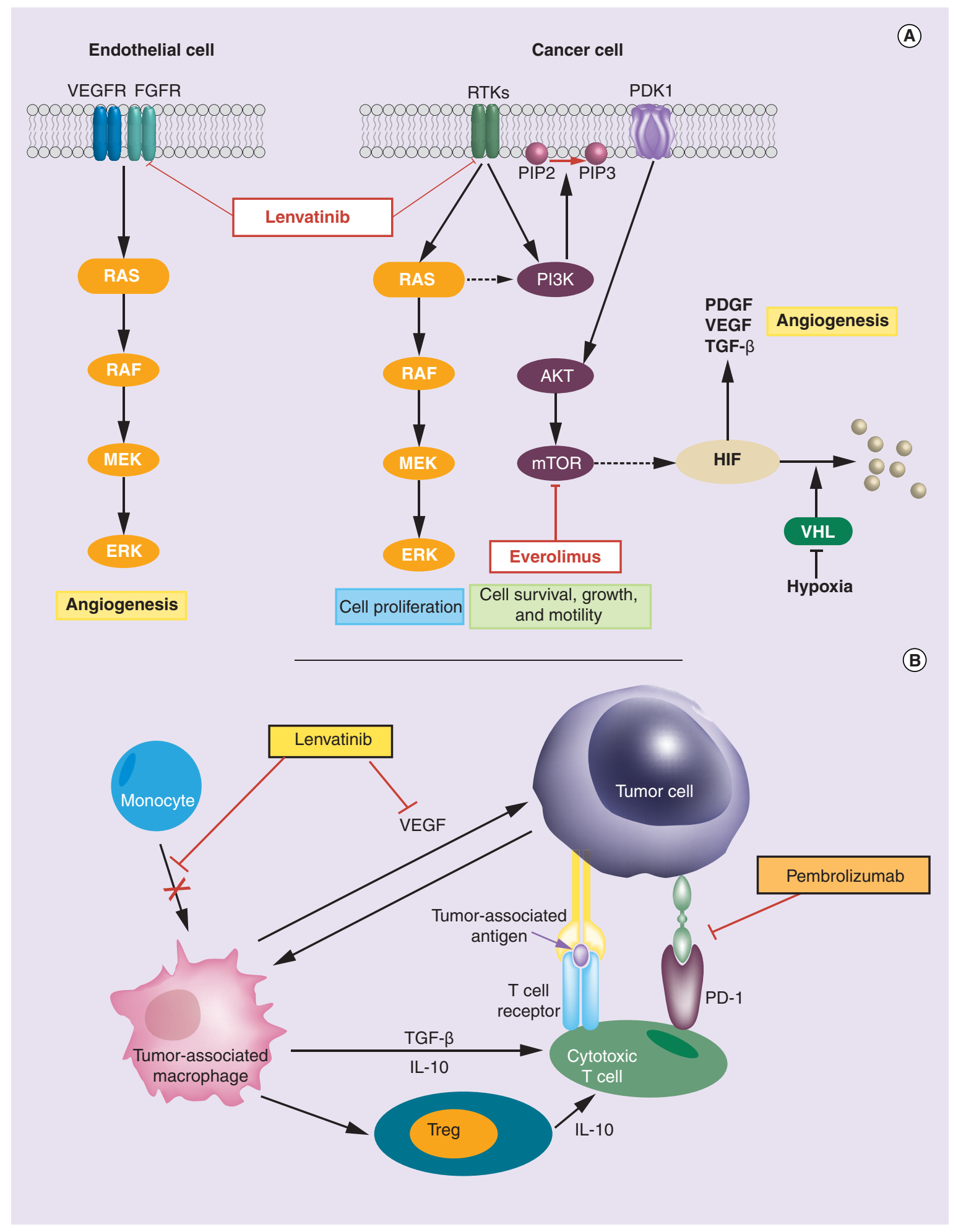

Figure 1. The mechanism of action of lenvatinib with its combination partners. Mechanisms of action of (A) lenvatinib plus everolimus and (B) lenvatinib plus pembrolizumab combination therapies.

IL: Interleukin; VHL: von Hippel-Lindau.

(A) Reproduced with permission from [53].

(B) Adapted with permission from [55]. 
Preliminary results from a Phase $\mathrm{Ib} / \mathrm{II}$ study $(\mathrm{n}=13)$ evaluating the combination of lenvatinib plus pembrolizumab showed promising activity in a range of solid tumor types [64]. Among eight patients with RCC enrolled in the Phase Ib part of the study, five achieved a partial response as their best overall response and three achieved stable disease. The most common treatment-emergent adverse events were decreased appetite, diarrhea and fatigue (each $n=9$ ), and hypertension, hypothyroidism and nausea (each $n=8$ ). Generally, toxicities were managed with dose modifications [64].

An analysis of the RCC cohort of patients enrolled across both phases of the study $(n=30)$ strengthened the promising antitumor activity and acceptable safety profile of lenvatinib plus pembrolizumab [65]. The objective response rate (ORR) was $63.3 \%$ for all patients in the RCC cohort, and $83.3 \%$ in the first-line treatment setting [65]. Responses were seen regardless of prior treatment and PD-L1 status [65]. Toxicities (most commonly diarrhea, fatigue, hypothyroidism, nausea and stomatitis) were manageable with lenvatinib dose interruption and/or modification [65]. An updated analysis of the RCC cohort in this ongoing study recently reported an ORR of 70.0\%, with a median duration of response of 18.4 months [66]. The combination of lenvatinib plus pembrolizumab has been granted breakthrough therapy designation by the US FDA for patients with advanced or metastatic RCC [67]. Because a significant unmet medical need remains for more effective treatment options with manageable safety profiles for patients with aRCC, these combination therapies are the focus of the CLEAR study.

\section{The CLEAR study}

Here, we describe the design and rationale of the CLEAR study (NCT02811861; EudraCT 2016-000916-14), a multicenter, randomized, open-label and Phase III trial, designed to evaluate whether the combination of two targeted therapies (lenvatinib plus everolimus) or the combination of one targeted therapy plus a PD-1 inhibitor (lenvatinib plus pembrolizumab) would be superior to standard first-line therapy with single-agent sunitinib in patients with advanced or metastatic RCC. The study is funded by Eisai Inc., with study support also provided by Merck \& Co., Inc.

\section{Objective}

The primary objective of this study is to demonstrate superiority of first-line lenvatinib plus everolimus or lenvatinib plus pembrolizumab over sunitinib alone in prolonging PFS, assessed by independent imaging review (IIR) using Response Evaluation Criteria in Solid Tumors (RECIST) version (v) 1.1 in patients with aRCC. Secondary objectives are to compare ORR, OS, safety and tolerability, and health-related quality of life (HRQoL), as well as PFS, in patients on next-line therapy between treatment arms. The pharmacokinetic/pharmacodynamic relationship between exposure and efficacy, biomarkers and safety will also be explored. Exploratory end points include PFS in the lenvatinib plus pembrolizumab arm by immune-related RECIST (irRECIST) [68], duration of response, disease control rates, clinical benefit rates and the relationship between blood biomarkers and efficacy outcomes.

\section{Eligible patients}

Patients aged $\geq 18$ years should have histological or cytological confirmation of RCC with a clear cell component and documented evidence of advanced disease. Patients should also have at least one measurable target lesion according to RECIST v1.1, a Karnofsky performance status score of $\geq 70$, adequately controlled blood pressure (with or without the use of antihypertensive medications), and adequate renal, bone marrow, blood coagulation and liver function. Patients should not have received any prior systemic anticancer therapy for RCC. Patients also should not have received any radiation therapy within 21 days prior to the first dose of study drugs, except for palliative radiotherapy to bone lesions, which is permitted if completed 2 weeks prior to the start of study treatment. Patients with untreated CNS metastases or those who had received a live vaccine within 30 days of the planned first dose of study drug(s) are also excluded.

\section{Study design}

Patients are being recruited from Australia, Austria, Belgium, Canada, Czech Republic France, Germany, Greece, Ireland, Israel, Italy, Japan, the Netherlands, Poland, Russian Federation, South Korea, Spain, Switzerland, the UK and the USA. Eligible patients will be randomized centrally by an interactive voice- and web-response system in a 1:1:1 ratio to open-label treatment with oral lenvatinib $18 \mathrm{mg} /$ day plus oral everolimus $5 \mathrm{mg} /$ day, oral lenvatinib $20 \mathrm{mg} /$ day plus intravenous pembrolizumab $200 \mathrm{mg}$ every 3 weeks or oral sunitinib $50 \mathrm{mg} /$ day on a schedule of 4 weeks on and 2 weeks off (Figure 2). Randomization will be stratified according to the geographic region 


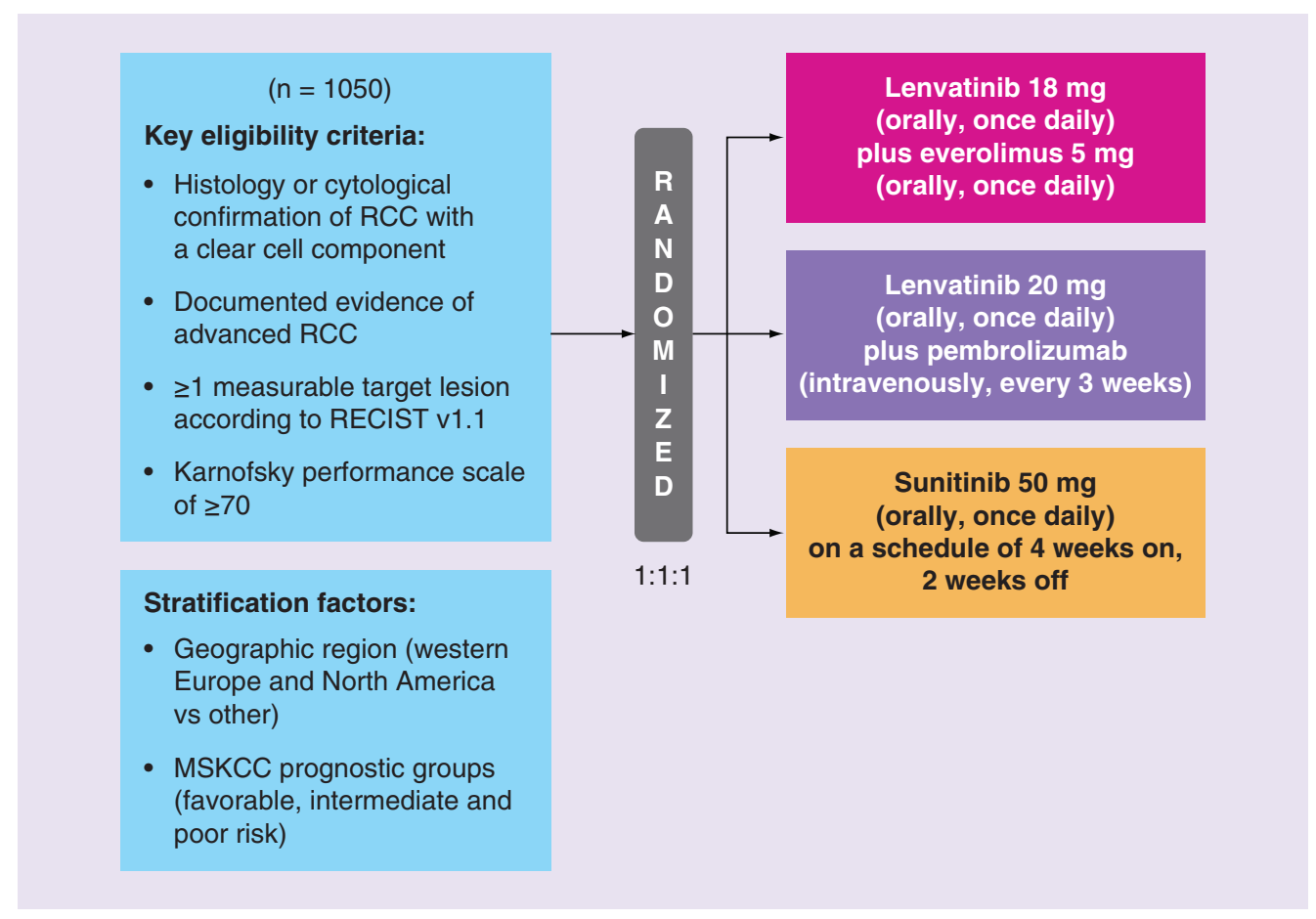

Figure 2. CLEAR study design.

MSKCC: Memorial Sloan Kettering Cancer Center; RCC: Renal cell carcinoma; RECIST v1.1: Response Evaluation Criteria In Solid Tumors version 1.1.

(western Europe and North America vs other) and Memorial Sloan Kettering Cancer Center (MSKCC) prognostic groups (favorable, intermediate or poor risk). Patients will continue to receive treatment until disease progression, unacceptable toxicity, patient request, withdrawal of consent, completion of 35 treatments with pembrolizumab or study termination. However, patients may continue treatment beyond initial RECIST v1.1-defined progression so long as an investigator-assessed clinical benefit is observed and the patient is tolerating the study drug. In this case, patients will discontinue study treatment on evidence of further progression and/or loss of clinical benefit.

\section{Outcome evaluations}

Tumor assessments, including computed tomography scans of the chest and computed tomography or magnetic resonance imaging scans of the abdomen, pelvis and other areas of known disease, will be performed every 8 weeks according to RECIST v1.1 by the investigator and sent to an imaging core laboratory for IIR. Patients randomized to the lenvatinib plus pembrolizumab treatment arm will also be assessed by IIR using immune-related RECIST. Tumor assessments will be performed at baseline, then every 8 weeks during treatment until disease progression or the start of another anticancer therapy for patients who discontinue study treatment without disease progression.

Safety assessments will consist of monitoring and recording of all adverse events from receipt of informed consent until the last visit; adverse events will be followed until 30 days after the last dose or until resolution, whichever comes first. Serious adverse events will be recorded from the receipt of informed consent until 120 days (or 30 days if the patient initiates new anticancer therapy) after the last dose, regardless of their relationship to the study drugs. Adverse events will be graded according to Common Terminology Criteria for Adverse Events, v4.03. Additionally, hematology and blood chemistry, urine values and vital sign variables will be regularly measured, and physical examinations, electrocardiography and multiple-gated acquisition scans or echocardiograms will be performed periodically. The HRQoL will be assessed at baseline, every 3 weeks, and at the time of withdrawal using several assessments: the Functional Assessment of Cancer Therapy Kidney Symptom Index-Disease-related Symptoms, the European Organisation for Research and Treatment of Cancer Quality of Life Questionnaire-C30 and the European Quality of Life (EuroQOL) EQ-SD-3L.

Blood samples will be collected from all lenvatinib recipients at baseline, every 2 weeks of treatment for the first two cycles and then every 3 weeks thereafter. Drug concentrations will be measured and analyzed using a population 
pharmacokinetic approach, and serum antidrug antibodies will also be measured for patients in the lenvatinib plus pembrolizumab treatment arm. A biomarker-discovery program is also associated with this clinical trial, focused on biomarker discovery and/or validation to identify potential biomarkers that may be useful to predict patient response, evaluate response-related and/or safety-related outcomes and for potential use in diagnostic development. Additionally, PD-L1 expression by immunohistochemistry will be evaluated.

\section{Statistical analyses}

The full analysis set (i.e., all randomized patients regardless of the treatment actually received) will be used for all efficacy analyses. In addition, the per protocol analysis set (i.e., patients who received $\geq 1$ dose of the study drug, had no major protocol deviations and had both baseline and $\geq 1$ postbaseline tumor assessments) will be used in secondary analysis of efficacy end points, and the safety analysis set (i.e., patients who received $\geq 1$ dose of the study drug) will be used for all safety analyses. The sample size was estimated based on the primary end point of PFS; approximately 1050 patients will be randomized. The study is designed to achieve at least $90 \%$ statistical power at two-sided $\alpha=0.0499$ to detect at a difference in at least one of the primary comparisons (lenvatinib plus everolimus vs sunitinib, or lenvatinib plus pembrolizumab vs sunitinib).

The primary efficacy analysis is planned for when approximately 551 PFS events have been observed among the three treatment arms and at least 368 events have been observed for the lenvatinib plus everolimus arm or the lenvatinib plus pembrolizumab arm and sunitinib arm. No comparative statistical analyses are planned between the combination arms. Median PFS will be estimated using the Kaplan-Meier method, with two-sided 95\% CIs, and the differences between treatment arms will be evaluated using the stratified log-rank test at two-sided $\alpha=0.05$, with geographical region and MSKCC prognostic groups as strata. A stratified Cox regression model will be used to estimate the HR and 95\% CI. For the secondary efficacy analysis, ORR will be determined with exact 95\% CIs using the Clopper-Pearson method, with the difference between treatment arms evaluated using a stratified Cochran-Mantel-Haenszel test. Median OS will be calculated using the same methods used in the primary PFS analysis. Additionally, the primary end point PFS and secondary end points ORR and OS will be tested using a parallel gatekeeping procedure to control the overall family-wise error rate.

\section{Discussion \& future perspective}

This is a pivotal Phase III study comparing outcomes with the current first-line treatment for aRCC (single-agent sunitinib) versus the combination of either two targeted therapies or one targeted therapy plus an immuno-oncology agent. Patients of all RCC risk groups will be included and stratified based on geographic region and MSKCC prognostic score. Results of the CLEAR study may reshape the approach to first-line treatment of aRCC by introducing a broader range of potential therapies. If the efficacy and safety of either of these combinations is confirmed, then one or two additional first-line treatment options will be available for patients with aRCC. In addition, exploratory analyses will investigate whether differences in biomarkers between TKI plus mTOR inhibitor and TKI plus immuno-oncology therapy combinations exist, and whether different patient populations may derive benefit from them - which could, in turn, inform patient selection. The treatment landscape for aRCC is rapidly evolving, and this study is one of several highly anticipated ongoing clinical trials to examine TKI-IO combination regimens. A key differentiator of the CLEAR study is that lenvatinib has demonstrated immune-modulating activity and has been shown to potentiate the antitumor activity of PD-1 signaling blockade in vivo [62]. These preclinical findings form the basis of a solid mechanistic rationale for the combination of these specific agents. Together with the promising anticancer activity observed with lenvatinib plus pembrolizumab in patients with aRCC in a Phase $\mathrm{Ib} / \mathrm{II}$ study [66], results of the CLEAR study are eagerly awaited.

There were several considerations taken into account during the clinical trial design stage. The first-line treatment paradigm is presently changing, particularly in patients with intermediate- and poor-risk features. However, sunitinib is likely to remain a reasonable first-line treatment option - particularly, but not exclusively, for patients with good risk features - so the use of sunitinib in the control arm of this study is justified, despite recent advancements in the first-line treatment setting. Additionally, tumor assessments will be performed using both investigator and independent reviews. Although independent review is often given more weight due to the lack of potential investigator bias, investigator-reviewed assessments are likely to be more reflective of real-world clinical practice, particularly when immunotherapy is involved [68]. Finally, whether the complete remission rate should be the standard outcome measure to evaluate medical treatment for patients with aRCC remains controversial. 
For targeted and cytokine therapies, the amount of tumor shrinkage typically correlates with survival outcomes, particularly if a threshold of $60 \%$ tumor shrinkage is reached [69].

Combinations of targeted agents may increase the amount of patients with at least $60 \%$ tumor shrinkage, thereby broadening the proportion of patients who may achieve an OS benefit from treatment. However, for checkpoint inhibitor immuno-oncology therapy, the role of tumor shrinkage as an outcome measure is still undefined. In a randomized, Phase III trial, the complete remission rate was higher with TKI plus immuno-oncology combination therapy (nivolumab plus ipilimumab) compared with single-agent TKI use (sunitinib) as first-line treatment for aRCC (9 vs $1 \%$, respectively), indicating a possible advantage with such a combination [18].

As expected with clinical trials of combination therapies, the safety and tolerability profile is of utmost importance to evaluate feasibility as part of the RCC treatment armamentarium. Data regarding HRQoL will also help inform on the usefulness of these therapies.

\section{Conclusion}

The CLEAR study will investigate the efficacy and tolerability of lenvatinib in combination with everolimus or pembrolizumab versus standard single-agent sunitinib as first-line therapy for aRCC. The results of this study will help define the roles of these novel combination therapies in the management of aRCC. If positive, the results of this study may change the treatment paradigm in first-line RCC to combination therapy, perhaps irrespective of the prognostic risk group.

\section{Executive summary}

\section{Background}

- Despite the availability of several first-line targeted treatment options for advanced renal cell carcinoma (RCC), current therapies are unsatisfactory.

- Approximately $20-30 \%$ of patients do not respond to initial therapy or progress within $\leq 3$ months, indicating resistance to molecular-targeted monotherapies.

Lenvatinib

- Lenvatinib is a potent, multiple-receptor, tyrosine kinase inhibitor that selectively targets VEGF receptors $1-3$, FGF receptors 1-4, PDGFR $\alpha$, KIT and RET.

- Lenvatinib in combination with everolimus is approved for the treatment of patients with advanced RCC after one prior VEGF-targeted therapy.

- In a Phase I study, lenvatinib in combination with pembrolizumab demonstrated promising antitumor activity in patients with RCC.

The CLEAR study

- The CLEAR study is a three-arm, Phase III clinical trial investigating the combination of lenvatinib plus everolimus and the combination of lenvatinib plus pembrolizumab versus sunitinib monotherapy for the first-line treatment of patients with advanced RCC.

- Eligible patients will have advanced RCC with histologically or cytologically confirmed clear cell component and no prior systemic anticancer therapy.

- The primary end point is progression-free survival, as assessed by independent imaging reviewers using Response Evaluation Criteria In Solid Tumors version 1.1.

- Results of this trial will help define the roles of combination therapies - targeted therapy combinations as well as targeted therapy plus immuno-oncology approaches - in the management of advanced RCC.

Supplementary data

An infographic accompanies this paper at the end of the references section. To download the infographic that accompanies this paper, please visit the journal website at: www.futuremedicine.com/doi/full/10.2217/fon-2018-0745

Financial \& competing interests disclosure

This study is funded by Eisai Inc., Woodcliff Lake, NJ, USA, and Merck \& Co., Inc., Kenilworth, NJ, USA. Editorial support with the preparation of this manuscript was provided by Marcel Kuttab, PharmD, of Oxford PharmaGenesis Inc., Newtown, PA, USA, with funding provided by Eisai Inc. 
V Grünwald reports grants, personal fees and nonfinancial support from Bristol-Myers Squibb, Merck Sharp \& Dohme and Novartis; personal fees and nonfinancial support from Roche and Ipsen; grants and personal fees from AstraZeneca and Pfizer; and personal fees from Eisai, EUSA Pharma and Cerulean.

T Powles reports grants/research support from Merck, Roche, AstraZeneca, Pfizer, Novartis and Exelixis; and has served as a consultant and received honoraria from Merck, Roche, AstraZeneca, Pfizer, Novartis and Exelixis.

TK Choueiri reports consulting/advisory boards fees from AstraZeneca, Alexion, Sanofi/Aventis, Bayer, BMS, Cerulean, Eisai, Foundation Medicine Inc., Exelixis, Genentech, Heron Therapeutics, Roche, GlaxoSmithKline, Merck, Novartis, Peloton, Pfizer, EMD Serono, Prometheus Labs, Corvus, Ipsen, Up-to-Date, NCCN, Analysis Group; reports grants/research support (institutional and personal) from AstraZeneca, Bayer, BMS, Cerulean, Eisai, Foundation Medicine Inc., Exelixis, Ipsen, Tracon, Genentech, Roche, Roche Products Limited, GlaxoSmithKline, Merck, Novartis, Peloton, Pfizer, Prometheus Labs, Corvus, Calithera, Analysis Group, Takeda; reports honoraria from AstraZeneca, Alexion, Sanofi/Aventis, Bayer, BMS, Cerulean, Eisai, Foundation Medicine Inc., Exelixis, Genentech, Roche, GlaxoSmithKline, Merck, Novartis, Peloton, Pfizer, EMD Serono, Prometheus Labs, Corvus, Ipsen, Up-toDate, NCCN, Analysis Group, NCCN, Michael J Hennessy Associates, L-path, Kidney Cancer Journal, Clinical Care Options, Platform Q, Navinata Healthcare, Harborside Press, American Society of Medical Oncology, NEJM, Lancet Oncology, and Heron Therapeutics.

TE Hutson served as a consultant and received honoraria from Aveo, BMS, Eisai, Exelixis, Novartis, and Pfizer; and has participated in speakers' bureaus for BMS, Eisai, Exelixis and Pfizer.

C Porta reports grants/research support from Pfizer; has served as a consultant for Novartis, Pfizer, BMS, Ipsen, Eisai, Jannsen, EUSA and Peloton; and has participated in speakers' bureaus for Novartis, Pfizer, BMS, Ipsen and Eisai.

M Eto reports grants/research support from Pfizer, Novartis, Astellas, Sanofi, Takeda, Lilly, Ono, and Nippon Kayaku; has served as a consultant for Novartis, BMS, and Pfizer; and has participated in speakers' bureaus for Pfizer, Novartis, Bayer, Astellas, Sanofi, Takeda, GSK, Ono and BMS.

CN Sternberg reports honoraria and consultancy from Novartis, Pfizer, Ipsen, Eisai, BMS, Roche, Bayer, and Merck Sharp \& Dohme Corp. C He, CE Dutcus, A Smith, L Dutta and K Mody are employees of Eisai. SY Rha has no conflicts to disclose. RJ Motzer reports grants/research support from Pfizer, Eisai, Exelixis, Genentech/Roche, Novartis and BMS; and has served as a consultant for Pfizer, Eisai, Exelixis, Merck, Incyte and Novartis.

The authors have no other relevant affiliations or financial involvement with any organization or entity with a financial interest in or financial conflict with the subject matter or materials discussed in the manuscript apart from those disclosed.

ClinicalTrials.gov: NCT02811861.

\section{Open access}

This work is licensed under the Attribution-NonCommercial-NoDerivatives 4.0 Unported License. To view a copy of this license, visit http://creativecommons.org/licenses/by-nc-nd/4.0/

\section{References}

Papers of special note have been highlighted as: $\bullet$ of interest; $\bullet \bullet$ of considerable interest

1. Bray F, Ferlay J, Soerjomataram I, Siegel RL, Torre LA, Jemal A. Global cancer statistics 2018: GLOBOCAN estimates of incidence and mortality worldwide for 36 cancers in 185 countries. CA Cancer J. Clin. 68(6), 394-424 (2018).

2. Ferlay J, Soerjomataram I, Dikshit R et al. Cancer incidence and mortality worldwide: sources, methods and major patterns in GLOBOCAN 2012. Int. J. Cancer 136(5), e359-e386 (2015).

3. American Cancer Society. Cancer facts \& figures 2018 (2018). www.cancer.org/content/dam/cancer-org/research/cancer-facts-and-statis tics/annual-cancer-facts-and-figures/2018/cancer-facts-and-figures-2018.pdf

4. Kabaria R, Klaassen Z, Terris MK. Renal cell carcinoma: links and risks. Int. J. Nephrol. Renovasc. Dis. 9, 45-52 (2016).

5. Znaor A, Lortet-Tieulent J, Laversanne M, Jemal A, Bray F. International variations and trends in renal cell carcinoma incidence and mortality. Eur. Urol. 67(3), 519-530 (2015).

6. Pal SK, Quinn DI. Differentiating mTOR inhibitors in renal cell carcinoma. Cancer Treat. Rev. 39(7), 709-719 (2013).

7. Choueiri TK, Motzer RJ. Systemic therapy for metastatic renal-cell carcinoma. N. Engl. J. Med. 376(4), 354-366 (2017).

8. Faivre S, Kroemer G, Raymond E. Current development of mTOR inhibitors as anticancer agents. Nat. Rev. Drug Discov. 5(8), 671-688 (2006).

9. Tsimafeyeu I, Demidov L, Stepanova E, Wynn N, Ta H. Overexpression of fibroblast growth factor receptors FGFR1 and FGFR2 in renal cell carcinoma. Scand. J. Urol. Nephrol. 45(3), 190-195 (2011).

10. Massari F, Ciccarese C, Santoni M et al. Targeting fibroblast growth factor receptor (FGFR) pathway in renal cell carcinoma. Expert Rev. Anticancer Ther. 15(12), 1367-1369 (2015).

- A useful primer for the role of FGF/receptor in renal cell carcinoma (RCC). 
11. Rasmuson T, Grankvist K, Jacobsen J, Ljungberg B. Impact of serum basic fibroblast growth factor on prognosis in human renal cell carcinoma. Eur. J. Cancer 37(17), 2199-2203 (2001).

12. Fukata $\mathrm{S}$, Inoue $\mathrm{K}, \mathrm{Kamada} \mathrm{M}$ et al. Levels of angiogenesis and expression of angiogenesis-related genes are prognostic for organ-specific metastasis of renal cell carcinoma. Cancer 103(5), 931-942 (2005).

13. Horstmann M, Merseburger AS, Von Der Heyde E et al. Correlation of bFGF expression in renal cell cancer with clinical and histopathological features by tissue microarray analysis and measurement of serum levels. J. Cancer Res. Clin. Oncol. 131(11), 715-722 (2005).

14. Tripathi A, Drake CG, Harshman LC. Harnessing the PD-1 pathway in renal cell carcinoma: current evidence and future directions. BioDrugs 28(6), 513-526 (2014).

15. Weinstock M, McDermott D. Targeting PD-1/PD-L1 in the treatment of metastatic renal cell carcinoma. Ther. Adv. Urol. 7(6), 365-377 (2015).

16. Zhu J, Armstrong AJ, Friedlander TW et al. Biomarkers of immunotherapy in urothelial and renal cell carcinoma: PD-L1, tumor mutational burden, and beyond. J. Immunother. Cancer 6(1), 4 (2018).

- A comprehensive summary of the utility of PD-L1 and other biomarkers for immunotherapy in RCC.

17. Motzer RJ, Escudier B, McDermott DF et al. Nivolumab versus everolimus in advanced renal-cell carcinoma. N. Engl. J. Med. 373(19), 1803-1813 (2015).

18. Motzer RJ, Tannir NM, McDermott DF et al. Nivolumab plus ipilimumab versus sunitinib in advanced renal-cell carcinoma. $N$. Engl. J. Med. 378(14), 1277-1290 (2018).

-• Results of this study showed an overall survival benefit with a combination regimen over a monotherapy for first-line therapy of advanced RCC.

19. Escudier B, Porta C, Schmidinger M et al. Renal cell carcinoma: ESMO Clinical Practice Guidelines for diagnosis, treatment and follow-up. Ann. Oncol. 27(Suppl. 5), v58-v68 (2016).

20. National Comprehensive Cancer Network Clinical Practice Guidelines in Oncology (NCCN Guidelines ${ }^{\circledR}$ ). Kidney Cancer. Version 4.2018 (2018). www.nccn.org/professionals/physician_gls/pdf/kidney.pdf

21. Patard JJ, Pignot G, Escudier B et al. ICUD-EAU International Consultation on Kidney Cancer 2010: treatment of metastatic disease. Eur. Urol. 60(4), 684-690 (2011).

22. Choueiri TK, Halabi S, Sanford BL et al. Cabozantinib versus sunitinib as initial targeted therapy for patients with metastatic renal cell carcinoma of poor or intermediate risk: the Alliance A031203 CABOSUN trial. J. Clin. Oncol. 35(6), 591-597 (2017).

23. US FDA. FDA approves nivolumab plus ipilimumab combination for intermediate or poor-risk advanced renal cell carcinoma (2018). www.fda.gov/drugs/informationondrugs/approveddrugs/ucm604685.htm

24. Ruiz-Morales JM, Heng DY. Cabozantinib in the treatment of advanced renal cell carcinoma: clinical trial evidence and experience. Ther. Adv. Urol. 8(6), 338-347 (2016).

25. Busch J, Seidel C, Weikert $\mathrm{S}$ et al. Intrinsic resistance to tyrosine kinase inhibitors is associated with poor clinical outcome in metastatic renal cell carcinoma. BMC Cancer 11, 295 (2011).

26. Heng DY, Mackenzie MJ, Vaishampayan UN et al. Primary anti-vascular endothelial growth factor (VEGF)-refractory metastatic renal cell carcinoma: clinical characteristics, risk factors, and subsequent therapy. Ann. Oncol. 23(6), 1549-1555 (2012).

27. Porta C, Sabbatini R, Procopio G, Paglino C, Galligioni E, Ortega C. Primary resistance to tyrosine kinase inhibitors in patients with advanced renal cell carcinoma: state-of-the-science. Expert Rev. Anticancer Ther. 12(12), 1571-1577 (2012).

28. Seidel C, Busch J, Weikert S et al. Progression free survival of first line vascular endothelial growth factor-targeted therapy is an important prognostic parameter in patients with metastatic renal cell carcinoma. Eur. J. Cancer 48(7), 1023-1030 (2012).

29. Morais C. Sunitinib resistance in renal cell carcinoma. J. Kidney Cancer VHL 1(1), 1-11 (2014).

30. Lim SH, Hwang IG, Ji JH et al. Intrinsic resistance to sunitinib in patients with metastatic renal cell carcinoma. Asia Pac. J. Clin. Oncol. 13(1), 61-67 (2017).

31. Sonpavde G, Willey CD, Sudarshan S. Fibroblast growth factor receptors as therapeutic targets in clear-cell renal cell carcinoma. Expert Opin. Investig. Drugs 23(3), 305-315 (2014).

32. Malouf GG, Flippot R, Khayat D. Therapeutic strategies for patients with metastatic renal cell carcinoma in whom first-line vascular endothelial growth factor receptor-directed therapies fail. J. Oncol. Pract. 12(5), 412-420 (2016).

33. Bukowski RM, Kabbinavar FF, Figlin RA et al. Randomized Phase II study of erlotinib combined with bevacizumab compared with bevacizumab alone in metastatic renal cell cancer. J. Clin. Oncol. 25(29), 4536-4541 (2007).

34. Feldman DR, Baum MS, Ginsberg MS et al. Phase I trial of bevacizumab plus escalated doses of sunitinib in patients with metastatic renal cell carcinoma. J. Clin. Oncol. 27(9), 1432-1439 (2009).

35. Hainsworth JD, Spigel DR, Burris HA 3rd, Waterhouse D, Clark BL, Whorf R. Phase II trial of bevacizumab and everolimus in patients with advanced renal cell carcinoma. J. Clin. Oncol. 28(13), 2131-2136 (2010). 
36. Négrier S, Gravis G, Pérol D et al. Temsirolimus and bevacizumab, or sunitinib, or interferon alfa and bevacizumab for patients with advanced renal cell carcinoma (TORAVA): a randomised Phase II trial. Lancet Oncol. 12(7), 673-680 (2011).

37. Graves A, Hessamodini H, Wong G, Lim WH. Metastatic renal cell carcinoma: update on epidemiology, genetics, and therapeutic modalities. Immunotargets Ther. 2, 73-90 (2013).

38. Ravaud A, Barrios CH, Alekseev BY et al. Randomized Phase II study of first-line everolimus plus bevacizumab (E+B) versus interferon $\alpha-2$ a plus bevacizumab (I+B) in patients (pts) with metastatic renal cell carcinoma (mRCC): record-2 final overall survival (OS) and safety results [abstract]. J. Clin. Oncol. 31(15 Suppl.), Abstract 4576 (2013).

39. Powles T, Foreshew SJ, Shamash $\mathrm{J}$ et al. A Phase Ib study investigating the combination of everolimus and dovitinib in vascular endothelial growth factor refractory clear cell renal cancer. Eur. J. Cancer 50(12), 2057-2064 (2014).

40. Kanesvaran R, Watt K, Turnbull JD, Armstrong AJ, Wolkowiez MC, George DJ. A single-arm Phase Ib study of everolimus and sunitinib in patients with advanced renal cell carcinoma. Clin. Genitourin. Cancer 13(4), 319-327 (2015).

41. Motzer RJ, Hutson TE, Glen $\mathrm{H}$ et al. Lenvatinib, everolimus, and the combination in patients with metastatic renal cell carcinoma: a randomised, Phase 2, open-label, multicentre trial. Lancet Oncol. 16(15), 1473-1482 (2015).

-• The pivotal Phase II trial leading to lenvatinib's first approval in advanced RCC, in combination with everolimus.

42. Motzer RJ, Powles T, Atkins MB et al. IMmotion151: a randomized Phase III study of atezolizumab plus bevacizumab vs sunitinib in untreated metastatic renal cell carcinoma (mRCC) [abstract]. J. Clin. Oncol. 36 (6 Suppl.), 578 (2018).

43. Motzer RJ, Penkov K, Haanen JB et al. JAVELIN renal 101: a randomized, Phase III study of avelumab + axitinib vs sunitinib as first-line treatment of advanced renal cell carcinoma (aRCC) [abstract]. Ann. Oncol. 29(Suppl. 8), mdy424.036 (2018).

44. Clinical Trials Database: NCT02853331. https://clinicaltrials.gov/ct2/show/NCT02853331

45. Merck's KEYTRUDA ${ }^{\circledR}$ (pembrolizumab) in combination with Pfizer's Inlyta ${ }^{\circledR}$ (axitinib) significantly improved overall survival (OS) and progression-free survival (PFS) as first-line therapy for advanced or metastatic renal cell carcinoma (2018).

https://investors.merck.com/news/press-release-details/2018/Mercks-KEYTRUDA-pembrolizumab-in-Combination-with-Pfizers-Inly ta-axitinib-Significantly-Improved-Overall-Survival-OS-and-Progression-f ree-Survival-PFS-as-First-Line-Therapy-for-Advanced-or -Metastatic-Renal-Cell-Carcinoma/default.aspx

46. Clinical Trials Database: NCT03141177. https://clinicaltrials.gov/ct2/show/NCT03141177

47. Matsui J, Funahashi Y, Uenaka T, Watanabe T, Tsuruoka A, Asada M. Multi-kinase inhibitor E7080 suppresses lymph node and lung metastases of human mammary breast tumor MDA-MB-231 via inhibition of vascular endothelial growth factor-receptor (VEGF-R) 2 and VEGF-R3 kinase. Clin. Cancer Res. 14(17), 5459-5465 (2008).

48. Matsui J, Yamamoto Y, Funahashi Y et al. E7080, a novel inhibitor that targets multiple kinases, has potent antitumor activities against stem cell factor producing human small cell lung cancer H146, based on angiogenesis inhibition. Int. J. Cancer 122(3), 664-671 (2008).

49. Okamoto K, Kodama K, Takase K et al. Antitumor activities of the targeted multi-tyrosine kinase inhibitor lenvatinib (E7080) against RET gene fusion-driven tumor models. Cancer Lett. 340(1), 97-103 (2013).

50. Yamamoto Y, Matsui J, Matsushima T et al. Lenvatinib, an angiogenesis inhibitor targeting VEGFR/FGFR, shows broad antitumor activity in human tumor xenograft models associated with microvessel density and pericyte coverage. Vasc. Cell 6, 18 (2014).

51. Matsuki M, Adachi Y, Ozawa Y et al. Targeting of tumor growth and angiogenesis underlies the enhanced antitumor activity of lenvatinib in combination with everolimus. Cancer Sci. 108(4), 763-771 (2017).

52. Ichikawa K, Miyano SW, Adachi Y, Matsuki M, Okamoto K, Matsui J. Lenvatinib suppresses angiogenesis through the inhibition of both the VEGFR and FGFR signaling pathways. Glob. J. Cancer Ther. 2(1), 019-025 (2016).

53. Leonetti A, Leonardi F, Bersanelli M, Buti S. Clinical use of lenvatinib in combination with everolimus for the treatment of advanced renal cell carcinoma. Ther. Clin. Risk Manag. 13, 799-806 (2017).

54. Boss DS, Glen H, Beijnen JH et al. A Phase I study of E7080, a multitargeted tyrosine kinase inhibitor, in patients with advanced solid tumours. Br. J. Cancer 106(10), 1598-1604 (2012).

55. Kudo M. Immuno-oncology in hepatocellular carcinoma: 2017 update. Oncology 93(Suppl. 1), 147-159 (2017).

56. Molina AM, Hutson TE, Larkin J et al. A Phase Ib clinical trial of the multi-targeted tyrosine kinase inhibitor lenvatinib (E7080) in combination with everolimus for treatment of metastatic renal cell carcinoma (RCC). Cancer Chemother. Pharmacol. 73(1), 181-189 (2014).

57. Inman S. FDA grants priority review to lenvatinib/everolimus combo for RCC (2016). www.onclive.com/web-exclusives/fda-grants-priority-review-to-lenvatinib-everolimus-combo-for-rcc

58. Lenvima (lenvatinib) [prescribing information]. Eisai Inc., Woodcliff Lake, NJ, USA (2018).

59. Lenvima $4 \mathrm{mg}$ hard capsules [summary of product characteristics]. Eisai Europe Limited, Hertfordshire, UK (2016).

60. Clinical Trials Database: NCT03173560. https://clinicaltrials.gov/ct2/show/NCT03173560

61. Ott PA, Hodi FS, Buchbinder EI. Inhibition of immune checkpoints and vascular endothelial growth factor as combination therapy for metastatic melanoma: an overview of rationale, preclinical evidence, and initial clinical data. Front. Oncol. 5, 202 (2015). 
62. Kimura T, Kato Y, Ozawa Y et al. Immunomodulatory activity of lenvatinib contributes to antitumor activity in the Hepa1-6 hepatocellular carcinoma model. Cancer Sci. 109(12), 3993-4002 (2018).

63. Kato Y, Albu D, Matsuki M et al. Upregulation of memory $\mathrm{T}$ cell population and shifting to Th1 response by lenvatinib potentiate antitumor activity of PD-1 signaling blockade: lenvatinib and PD-1 mAb combination. Presented at: The American Association of Cancer Research (AACR) Annual Meeting. Washington, DC, USA, 1-5 April 2017.

64. Taylor M, Dutcus CE, Schmidt E et al. A Phase Ib trial of lenvatinib (LEN) plus pembrolizumab (PEM) in patients with selected solid tumors [abstract]. Ann. Oncol. 27(Suppl. 6), Abstract 776PD (2016).

65. Lee C-H, Makker V, Rasco D et al. A Phase Ib/II trial of lenvatinib + pembrolizumab in patients with renal cell carcinoma. Presented at: The European Society for Medical Oncology (ESMO) Annual Congress. Madrid, Spain, 9 September 2017. Lee C-H, Makker V, Rasco $\mathrm{D}$ et al. Lenvatinib + pembrolizumab in patients with renal cell carcinoma: updated results. Presented at: The American Society of Clinical Oncology (ASCO) Annual Meeting. Chicago, IL, USA, 1-5 June 2018.

- Preliminary evidence of lenvatinib plus pembrolizumab antitumor activity in patients with advanced RCC was observed in this Phase Ib/II study.

67. Eisai and Merck receive breakthrough therapy designation from FDA for LENVIMA ${ }^{\circledR}$ (lenvatinib mesylate) and KEYTRUDA ${ }^{\circledR}$ (pembrolizumab) as combination therapy for advanced and/or metastatic renal cell carcinoma (2018). http://investors.merck.com/news/press-release-details/2018/Eisai-and-Merck-Receive-Breakthrough-Therapy-Desig nation-f rom-FDA-for-LENVIMA-lenvatinib-mesylate-and-KEYTRUDA-pembrolizumab-as-Combination-Therapy-for-Advanced-a nd-or-Metastatic-Renal-Cell-Carcinoma-/default.aspx

68. Wolchok JD, Hoos A, O'Day $S$ et al. Guidelines for the evaluation of immune therapy activity in solid tumors: immune-related response criteria. Clin. Cancer Res. 15(23), 7412-7420 (2009).

69. Grünwald V, Mckay RR, Krajewski KM et al. Depth of remission is a prognostic factor for survival in patients with metastatic renal cell carcinoma. Eur. Urol. 67(5), 952-958 (2015). 

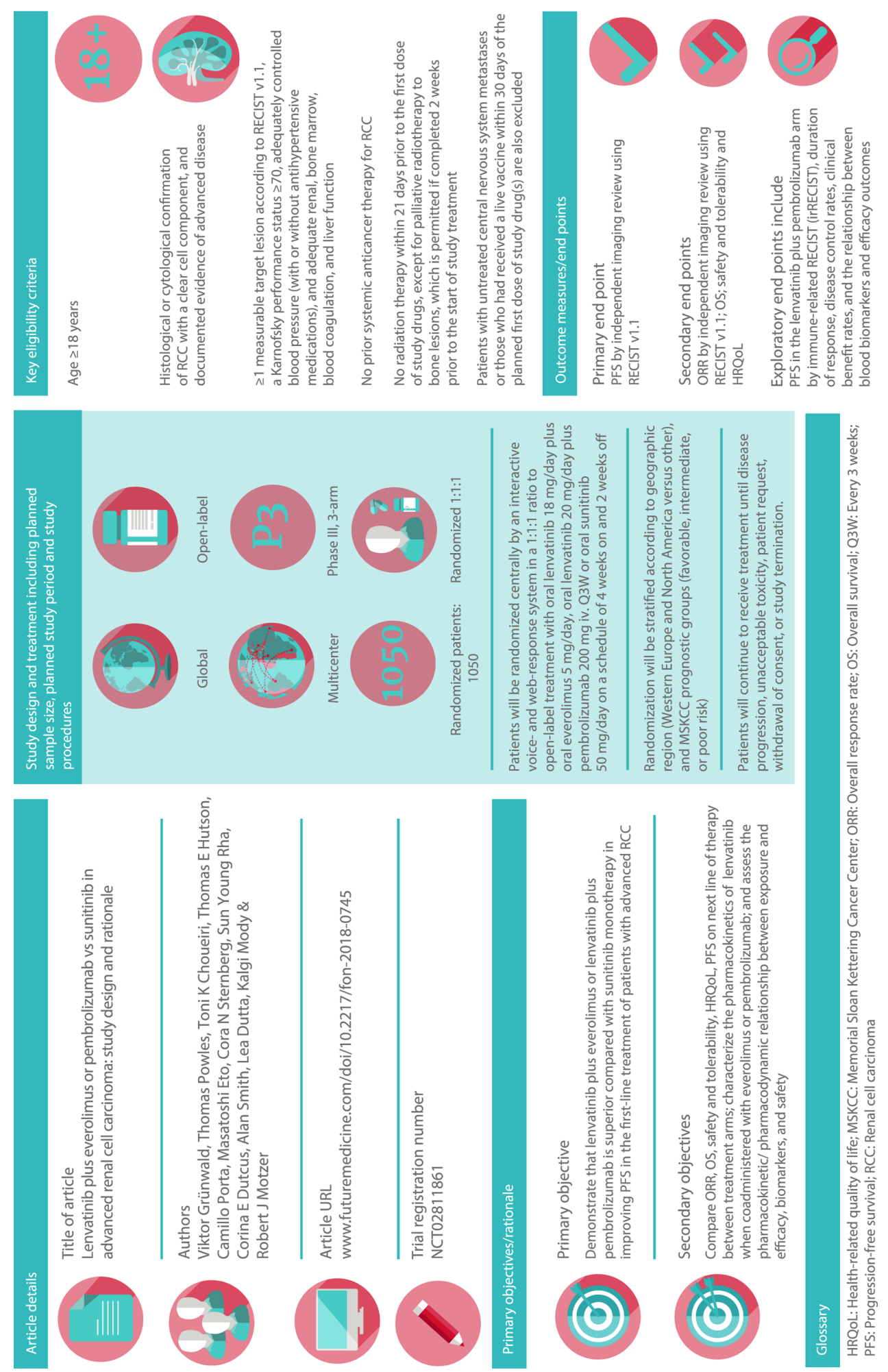